\title{
A CFD study on the evaporative cooling of a water droplet located in a duct
}

\author{
Aref Khorammi ${ }^{1}$, Amin Emamian ${ }^{2}$ and Sajjad Karimnejad ${ }^{2}$ \\ ${ }^{1}$ Department of Mechanical Engineering, University of Bojnord, Bojnord, Iran, e-mail: aref.khoramii@ gmail.com; \\ ${ }^{2}$ Mechanical Engineering Department, Shahrood University of Technology, Shahrood, Iran, email: \\ karimnejad.sajjad@gmail.com; aminemamian70@gmail.com
}

Article type: Original Scientific Article

\begin{tabular}{l} 
Article Info \\
\hline Article history: \\
Received April 2, 2021 \\
Revised June 13, 2021 \\
Accepted June 16, 2021 \\
\hline
\end{tabular}

\section{Keywords:}

Heat transfer, Droplet,

Air-water contact,

Evaporation,

Cooling.

\section{Corresponding Author:}

Sajjad. Karimnejad,

Mechanical Engineering Department, Shahrood University of Technology, Shahrood, Iran.

Email: karimnejad.sajjad@gmail.com

\begin{abstract}
The current numerical study aimed for analayzing heat transfer of a spherical water droplet placed in a rectangular duct. The problem particularly deals with air-cooling of the droplet. The computational fluid dynamic technique is used to treat the problem. In order to study the problem, the effect of evaporation on the droplet's heat transfer is taken into consideration to make the problem more realistic. Moreover, various parameters like the inlet velocity and the temperature of the upstream are numerically checked. It is found out that with involving evaporation, the mean temperature of the droplets increases in comparison with the case that there is no evaporation involved. The outcomes of the current study offer useful and deep insight into the evaporative cooling of a water droplet.
\end{abstract}

Copyright $(2020$ Regional Association for Security and crisis management and European centre for operational research. All rights reserved.

\section{Introduction}

The problem of multi-phase flow and particularly an air-water interface is seen in a wide variaty of natutal and engineering applications ranging from direct evaporative cooler to spray room to printers to human body (Mahdavi et al., 2020; Abed et al., 2020a, 2020b; Jia \& Qiu, 2003; Betelin et al., 2012; Semenov et al., 2011). More specifically, the presence of a droplet in a surrounding environment with different features is of great importance (Jia \& Qiu, 2003; Semenov et al., 201; Norouzi et al., 2019; Fisenko \& Khodyko, 2009), The problem can be even more complicated with the existence of heat transfer between two lquids and cooling phenomenon which is somewhat pratical. The driving potential for heat transfer is mainly attributed to the temperature gradient between the two parts of the environment and the droplet itself (Schlesinger et al., 2016).

Cuck et al. (1987) experimentally studied the thermal condition of a pan of water in a turbulent regime to measure evaporation rate. Liu et al. (2017) studied moisture and heat transfer of a deep air-buried duct and investigated charectristics of them along with condensation to shed ligh on underground construction. Pan et al. (2013) studied the mechanisms of water droplet evaporation in case of hydrophobic and superhydrophobic condition. In another study, Pan et al. (2014) moved toward surface wettability of the water droplet's transport mechanisms. Yang et al. (2014) employed Arbitrary-Lagrangian-Eulerian method to numerically tackle the evaporation problem of sessile droplet. Chen et al. (2016) numerically assessed the dynamics and evaporative cooling of a droplet which is impinged to a heated surface. Wei et al. (2017) hired a simplified computational fluid dynamic model to treat the problem of air-water direct contact. 
In the current study, authors has aimed for cooling a high-temperature spherical water droplet by means of air. The process takes place as air passess over a stationary droplet (water) located in an enclosure. The application of such problem can be seen in cooling towers and spraying. The schematic illustration of the problem and dimensions of the geometry are domenstrated in Figure 1 and Table 1, respectively. The effect of various parameters like inlet velocity, and upstream temperature on heat transfer is studied. More specifically, unlike most previous studies, the effect of evaporation is taken into consideration so that one can achieve a more realstic information for the under-study problem. Sophisticated verification test with previous studies are done to check the credibility of the results and acceptable agreement is seen. As for the numerical simulation, computer fluid dynamic (CFD) technique is hired in a three-dimensional problem and simulation is done using COMSOL software.

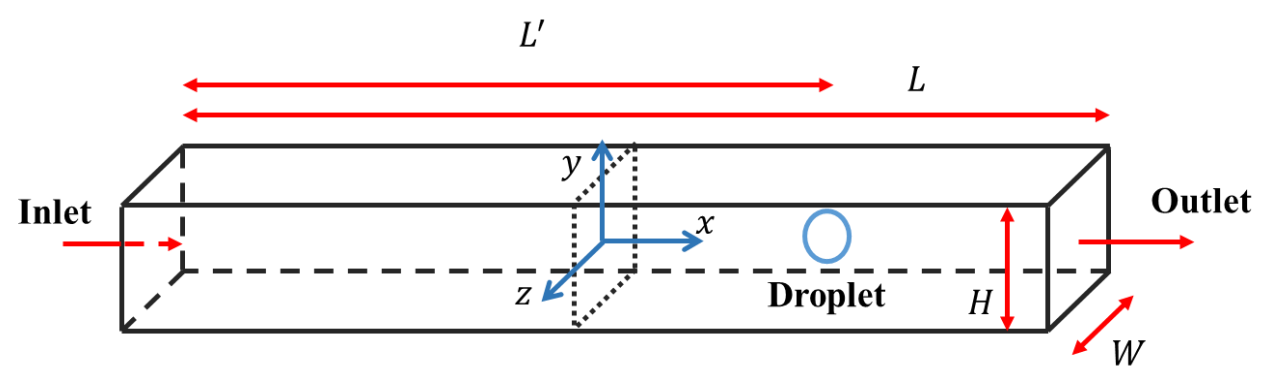

Figure 1. Schematic illustration of the under-study problem

Table 1. Corresponding dimensions of the geometry

\begin{tabular}{cccc}
\hline$L(m)$ & $L^{\prime}(m)$ & $H(m)$ & $W(m)$ \\
\hline 0.85 & 0.68 & 0.18 & 0.18 \\
\hline
\end{tabular}

The rest of the paper is organized as follows. Section 2 is responsible for the governing equations, following which verification test and important results are presented in section 3 . Finally, conclusion is stated in section 4.

\section{Governing equtions}

In this section the important relations and governing equation for the current study are presented. The turbulent flow, low Reynolds (Re) numbers, and $k-\varepsilon$ interface which can be used for fixed and time-dependent analyses are hired for studying single-phase and multi-phase flows at high Re numbers. The physics' interface is appropriate for incompressible flows, weakly compressible flows, and compressible flows at low Mach numbers (typically less than 0.3) (Semenov et al., 2011).

$$
\begin{aligned}
& \rho \nabla \cdot \boldsymbol{u}=0, \\
& \rho(\boldsymbol{u} \cdot \nabla) \boldsymbol{u}=\nabla \cdot[-p \boldsymbol{I}+\boldsymbol{K}]+\boldsymbol{F}, \\
& \rho(\boldsymbol{u} \cdot \nabla) k=\nabla \cdot\left[\left(\mu+\frac{\mu_{T}}{\sigma_{k}}\right) \nabla k\right]+P_{k}-\rho \epsilon, \\
& \rho(\boldsymbol{u} \cdot \nabla) \epsilon=\nabla \cdot\left[\left(\mu+\frac{\mu_{T}}{\sigma_{\epsilon}}\right) \nabla \epsilon\right]+C_{\epsilon 1} \frac{\epsilon}{k} P_{k}-C_{\epsilon 2} \rho \frac{\epsilon^{2}}{k} f_{\epsilon}, \\
& \nabla G \cdot \nabla G+\sigma_{w} G(\nabla \cdot \nabla G)=\left(1+2 \sigma_{w}\right) G^{4}, \\
& \quad \text { where } \\
& \boldsymbol{K}=\left(\mu+\mu_{T}\right)\left(\nabla \boldsymbol{u}+(\nabla \boldsymbol{u})^{T}\right), \\
& \mu_{T}=\rho C_{\mu} \frac{k^{2}}{\epsilon} f_{\mu}, \\
& P_{k}=\mu_{T}\left[\nabla \boldsymbol{u}:\left(\nabla \boldsymbol{u}+(\nabla \boldsymbol{u})^{T}\right)\right],
\end{aligned}
$$

where in Equations (1)-(8), $\rho$ stands for density, $\boldsymbol{u}$ is velocity vector, and $p$ refers to pressure. Also, $\boldsymbol{F}$ is the body force, $k$ is the turbulence kinetic energy $\left(\frac{3}{2}\left(U_{r e f} l_{T}\right)^{2}\right), U_{r e f}$ is the reference velocity which is equal to $U_{0}\left(U_{0}\right.$ is input velocity). $l_{T}$ is the turbulent intensity, $\mu, \mu_{T}$ are respectively the dynamic and turbulent 
viscosity, and $\epsilon$ is dissipation rate of turbulent kinetic energy $\left(C_{\mu}^{3 / 4} \frac{k^{\frac{3}{2}}}{L_{T}}\right)$, where $L_{T}$ is turbulence length scale, and $G$ is the reciprocal wall distance. The six constants $C_{\mu}, \sigma_{k}, \sigma_{\epsilon}, \sigma_{w}, C_{\epsilon 1}$, and $C_{\epsilon 2}$ are based on high Reynoldsnumber $k$ - $\epsilon$ models (Fan et al., 1993). The near-wall and low-Reynolds number function $f_{\epsilon}$ and $f_{\mu}$ depend on the physical properties and geometry of the problem (Fan et al., 1993; Semenov et al., 2011).

\subsection{Heat transfer in moist air}

The energy equation is used here for measuring heat transfer and particularly the cooling rate [6, 18].

$$
\underset{\text { where }}{\rho C_{p} \boldsymbol{u} \cdot \nabla T+\nabla \cdot \boldsymbol{q}=S,}
$$$$
\boldsymbol{q}=\kappa \nabla T,
$$

where $C_{p}$ is the specific heat capacity, and $T$ stands for temperature distribution. $\kappa$ is conductivity tensor, and $S$ is Heat source.

\subsection{Moisture transport in air}

To obtain the correct amount of water which is evaporated from the droplet into the air, the moisture transport in air interface is used (Wei et al., 2017). It should be pointed out that the amount of evaporated water here is negligible. As a result of that in this study, authors did not consider the volume change. It can be attributed to the fact that the considered time span is not long enough to make impact.

$$
\begin{aligned}
& M_{v} \boldsymbol{u} \cdot \nabla c_{v}+\nabla \cdot \mathbf{g}=G, \\
& \text { where } \\
& \mathbf{g}=-M_{v} D \nabla c_{v}, \\
& c_{v}=\phi c_{s a t},
\end{aligned}
$$

where $c_{v}$ is the vapor concentration, $c_{s a t}$ is the saturation concentration of vapor, $\phi$ is the relative humidity and $D$ is diffusion coefficient.

\section{Mesh independence}

In this section, independence of mesh is studied so that one can reach the desirable accuracy and calculation speed for the study. Table 2 presents the study done on the droplet's average temperature in various mesh numbers, 258900, 288800, 342500, and 486280, and corresponding errors. As one can easily deduce, considering both accuracy and calculation time, choosing mesh number three, 342500 , can be satisfactory.

Table 2. Mesh study for the under-study problem

\begin{tabular}{ccccc}
\hline Number of elements & 258900 & 288800 & 342500 & 486280 \\
\hline Average droplet & 71.82 & 73.38 & 74.79 & 74.90 \\
temperature $\left({ }^{\circ} \mathrm{C}\right)$ & 4.23 & 2.15 & 0.27 & 0.13 \\
Error $(\%)$ & &
\end{tabular}

\section{Results and discussion}

In this section the robustness and validity of the obtained data are first checked, then a quite detailed investigation on the air-cooling of the droplet is done.

In this section, the credibility of the simulation is first investigated with comparing the results with the work of Abdolnejad et al. (2018). So, the volume fraction of vapor against time step is considered as a verification test. As can be seen from Fig. 2, there is a good agreement between the results and it seems satisfactory. 


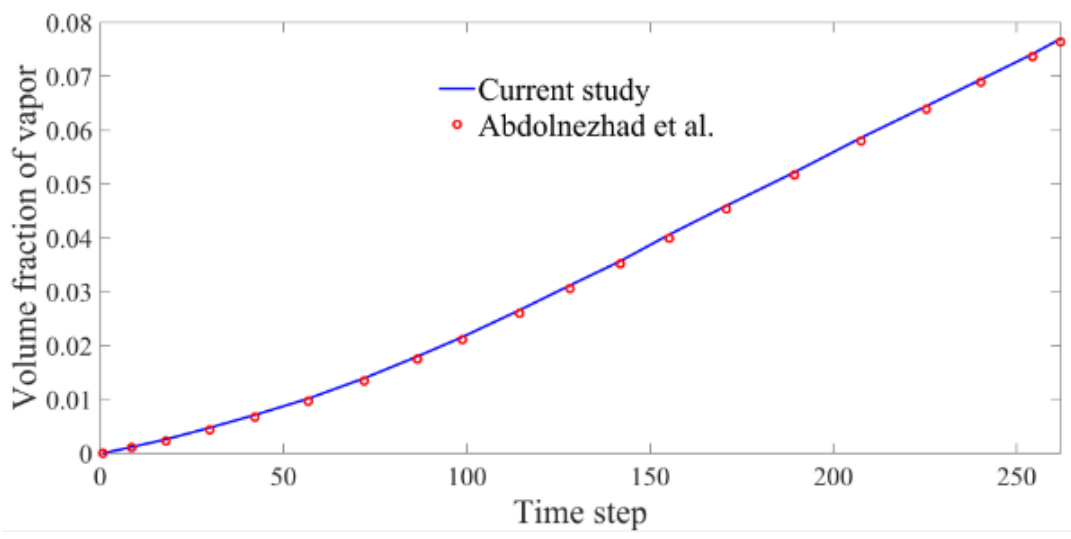

Figure 2. The verification test for the under-study problem.

Schematic illustration of the problem is as Fig. 1 and the corresponding information related to the geometry is stated in Table 1.

Figure 3 illustrates average temperature of water against time in two cases with and without evaporation. It should be mentioned that the upstream temperature $\left(T_{\text {upstr }}\right)$ equals $293 \mathrm{~K}$ and $U_{0}=2 \frac{\mathrm{m}}{\mathrm{s}}$. As can be seen from the figure two cases show the similar trend; however, the one with the presence of evaporation shows higher temperature. The gap between the two cases remains relatively unchanged after the first 5 minutes. Moreover, evaporation accounts for a decrease of about $13 \mathrm{~K}$ at the end of the simulation (see Fig. 3). It can be seen that the impact of evaporation is not negligible.

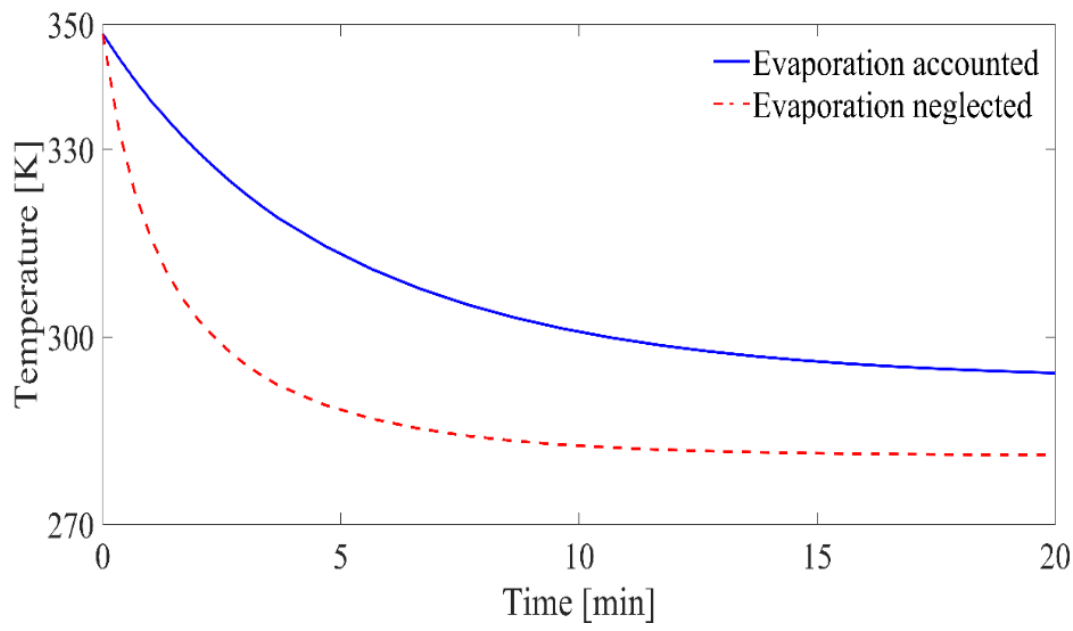

Figure 3. Average water temperature with and without evaporation accounted at $U_{0}=$

$$
2\left[\frac{m}{s}\right], T_{\text {usptr }}=293[K] .
$$

Following which, average temperature of droplet when evaporation is taken into consideration for various values of inlet velocity, namely 1,2, and $3 \mathrm{~m} / \mathrm{s}$, and $T_{\text {upstr }}=293 \mathrm{~K}$ is studied and results are shown in Fig. 4 . 


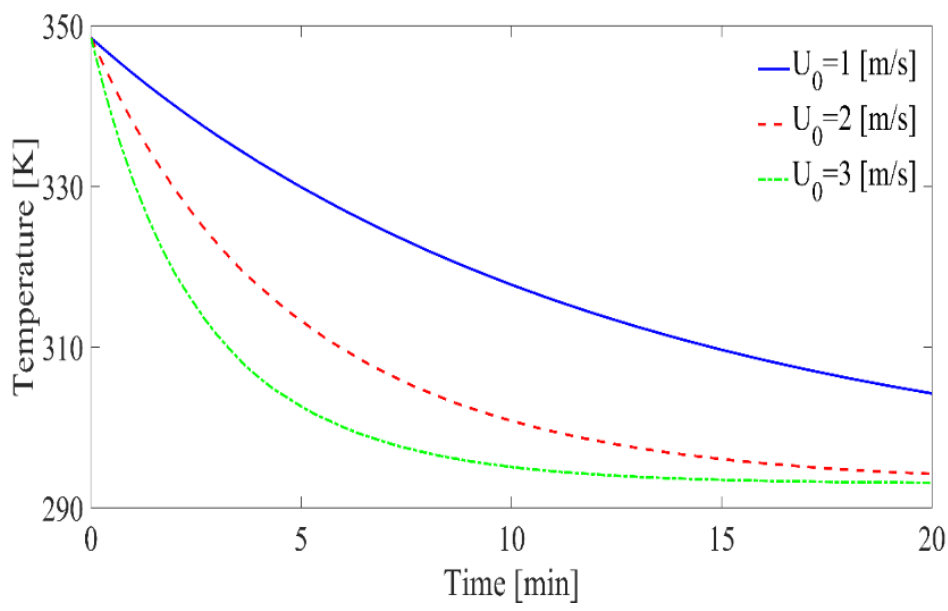

Figure 4. Average water temperature (evaporation accounted) for different value of the inlet velocity at $T_{\text {upstr }}=293[\mathrm{~K}]$.

Figure 5 shows the concentration and relative humidity at the symmetry plane. Close to the water surface, the relative humidity is about $100 \%$ as expected. One can deduce that due to the high temperature, air can absorb higher amount of water.

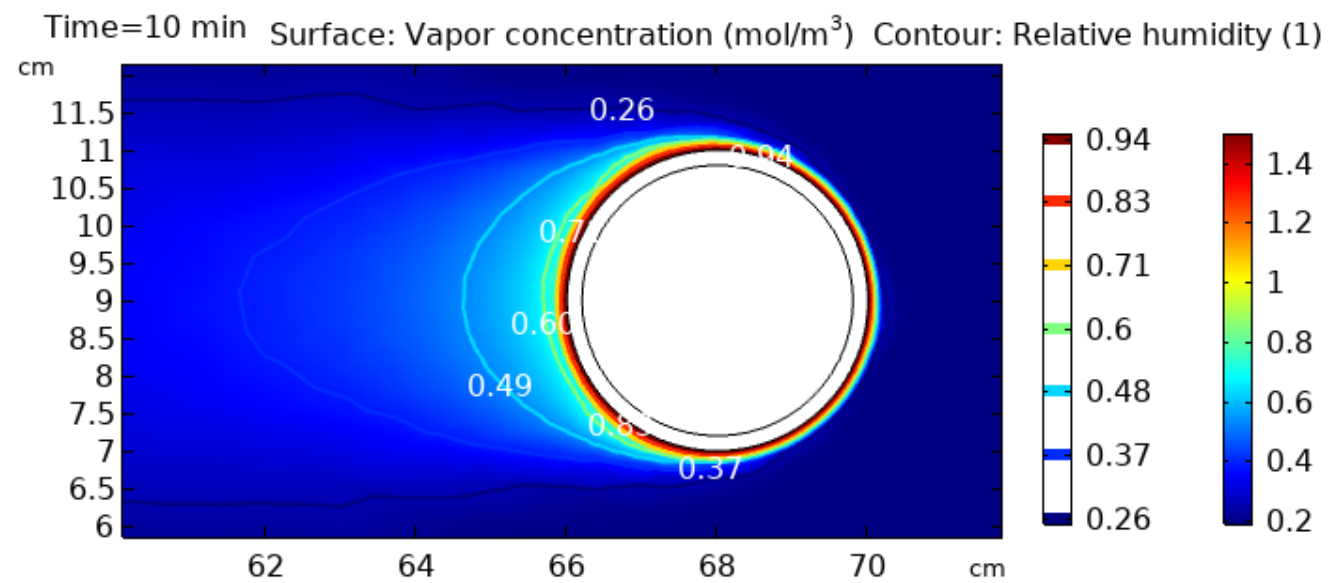

Figure 5. Concentration distribution and contour lines for the relative humidity.

Average water temperature of the droplet for various upstream temperatures, namely 288, 293, and $298 \mathrm{~K}$, at $U_{0}=2 \mathrm{~m} / \mathrm{s}$ is demonstrated in Fig. 6. As one can deduce, with an increase in the upstream temperature, the average temperature of the droplet also goes up. So, upstream temperature has its own effect on the rate of cooling. 


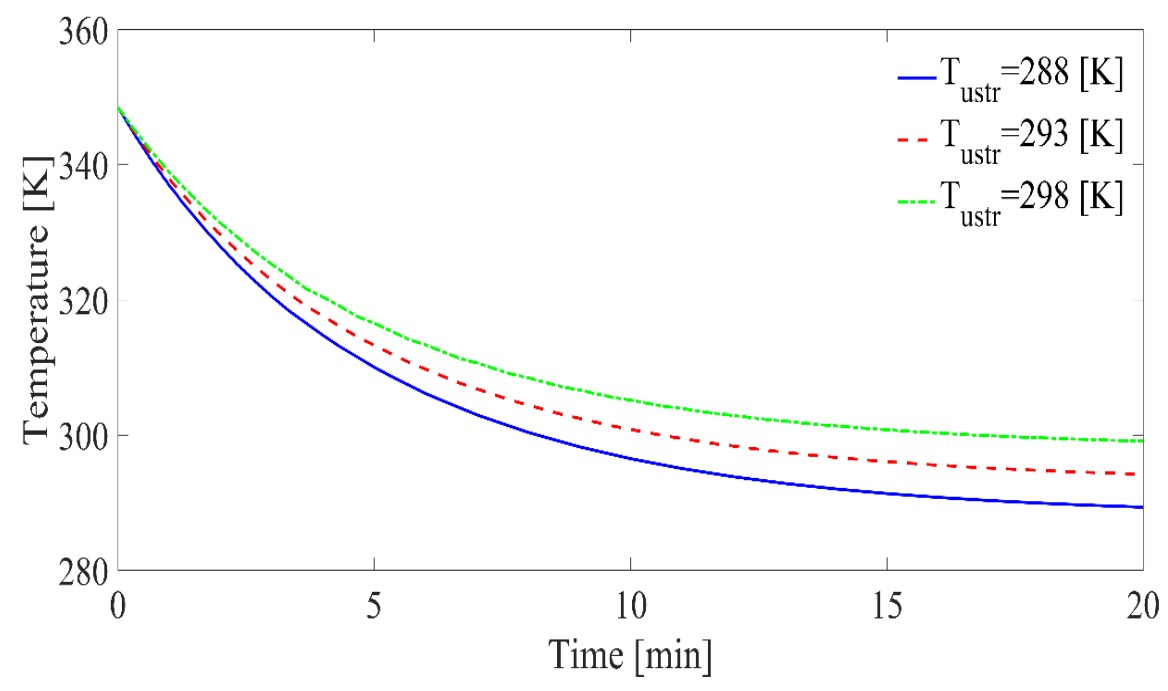

Figure 6. Average water temperature (evaporation accounted) for different values of $T_{\text {upstr }}$ at

$$
U_{0}=2\left[\frac{m}{s}\right] \text {. }
$$

\section{Conclusion}

In the current study, computational fluid dynamic technique is hired for studying the evaporative air-cooling of a Newtonian water drop in a duct. The importance of the problem is clear enough particularly for spraying applications and cooling towers. The authors tried making the problem more realistic with including the effect of evaporation. It is seen that through which, the mean temperature of the droplet increases. Also, the effect of inlet velocity and upstream temperature is investigated. It is seen that with increasing both of them, the average temperature of the droplet increases and decreases, respectively. The paper is useful for future studies when the temperature of droplet in the case of evaporation is of great importance.

\section{References}

Abdolnezhad, H., Nourozi, M., \& Emamian, A. (2018). 'Numerical investigation of a sedimenting droplet's falling dynamics and evaporation in a viscous fluid flow.' Internationa conference on applied research in science and engineering, 215-219.

Abed, A., Shcheklein, S., \& Pakhaluev, V. (2020a). Comparative study on steady and unsteady heat transfer analysis of a spherical element using air/water mist two-phase flow. Thermal Science, 20, 55-55.

Abed, A., Shcheklein, S., \& Pakhaluev, V. (2020b). An experimental investigation on the transient heat transfer characteristics using air/water droplets two-phase flow, in IOP Conference Series: Materials Science and Engineering, 791(1), 012001.

Arpaci, V.S., \& Larsen, P.S. (1984). Convection heat transfer. Prentice Hall.

Betelin, V., Smirnov, N., Nikitin, V., Dushin, V., Kushnirenko, A., \& Nerchenko, V. (2012). Evaporation and ignition of droplets in combustion chambers modeling and simulation. Acta Astronautica, 70, 23-35.

Chen, J.-N., Zhang, Z., Xu, R.N., Ouyang, X.L., \& Jiang, P.X. (2016). Numerical investigation of the flow dynamics and evaporative cooling of water droplets impinging onto heated surfaces: an effective approach to identify spray cooling mechanisms. Langmuir, 32(36), 9135-9155.

Chuck, W., \& Sparrow, E.M. (1987). Evaporative mass transfer in turbulent forced convection duct flows. International journal of heat and mass transfer, 30(2), 215-222.

Fan, S., Lakshminarayana, B., \& Barnett, M. (1993). Low-Reynolds-number k-epsilon model for unsteady turbulent boundary-layer flows. AIAA journal, 31(10), 1777-1784.

Fisenko, S. P. \& Khodyko, J. A. (2009). Low pressure evaporative cooling of micron-sized droplets of solutions and its novel applications. International journal of heat and mass transfer, 52(16), 3842-3849.

Jia, W., \& Qiu, H.H. (2003). Experimental investigation of droplet dynamics and heat transfer in spray cooling. 
Experimental Thermal and Fluid Science, 27(7), 829-838.

Liu, Y., Liu, S., \& Zhang, H. (2017). Numerical simulation of heat and moisture transfer in deep air buried. Procedia Engineering, 205, 1927-1933.

Mahdavi, M., Sharifpur, M. \& Meyer, J. P. (2020). Fluid flow and heat transfer analysis of nanofluid jet cooling on a hot surface with various roughness. International Communications in Heat and Mass Transfer, 118, 104842 .

Norouzi, M., Abdolnezhad, H., \& Mandani, S. (2019). An experimental investigation on inertia motion and deformation of Boger drops falling through Newtonian media. Meccanica, 54(3), 473-490.

Pan, Z., Dash, S., Weibel, J.A., \& Garimella, S.V. (2013). Assessment of water droplet evaporation mechanisms on hydrophobic and superhydrophobic substrates. Langmuir, 29(51), 15831-15841.

Pan, Z., Weibel, J.A., \& Garimella, S.V. (2014). Influence of surface wettability on transport mechanisms governing water droplet evaporation. Langmuir, 30(32), 9726-9730.

Schlesinger, D., Sellberg, J.A., Nilsson, A., \& Pettersson, L.G. (2016). Evaporative cooling of microscopic water droplets in vacuo: Molecular dynamics simulations and kinetic gas theory. The Journal of Chemical Physics, 144(12), 124-135.

Semenov, S., Starov, V., Velarde, M., \& Rubio, R. (2011). Droplets evaporation: Problems and solutions. The European Physical Journal Special Topics, 197(1), 265-271.

Wei, X., Duan, B., Zhang, X., Zhao, Y., Yu, M., \& Zheng, Y. (2017). Numerical simulation of heat and mass transfer in air-water direct contact using computational fluid dynamics. Procedia Engineering, 205, 25372544.

Yang, K., Hong, F., \& Cheng, P. (2014). A fully coupled numerical simulation of sessile droplet evaporation using Arbitrary Lagrangian-Eulerian formulation. International Journal of Heat and Mass Transfer, 70, 409-420. 